\title{
RIESZ BASIS PROPERTY AND EXPONENTIAL STABILITY OF CONTROLLED EULER-BERNOULLI BEAM EQUATIONS WITH VARIABLE COEFFICIENTS*
}

\author{
BAO-ZHU GUO ${ }^{\dagger}$
}

\begin{abstract}
This paper studies the basis property and the stability of a distributed system described by a nonuniform Euler-Bernoulli beam equation under linear boundary feedback control. It is shown that there is a sequence of generalized eigenfunctions of the system, which forms a Riesz basis for the state Hilbert space. The asymptotic distribution of eigenvalues, the spectrumdetermined growth condition, and the exponential stability are concluded. The results are applied to a nonuniform beam equation with viscous damping of variable coefficient as a generalization of existing results for the uniform beam.
\end{abstract}

Key words. beam equation, variable coefficients, asymptotic analysis, Riesz basis, stability

AMS subject classifications. 93C20, 93D15, 35B35, 35P10

PII. S0363012900372519

1. Introduction. The Riesz basis property, meaning that the generalized eigenvectors of the system form an unconditional basis for the state Hilbert space, is one of the fundamental properties of a linear vibrating system. The establishment of the basis property will naturally lead to solutions to such problems as the spectrumdetermined growth condition and the exponential stability for infinite dimensional systems. Unfortunately, verification of the Riesz basis generation is challenging even for extensively studied systems such as Euler-Bernoulli beam equations. Recently, a new approach has been suggested [1] to obtain a complete solution to the basis property of the following uniform Euler-Bernoulli beam equation under linear boundary feedback control:

$$
\left\{\begin{array}{l}
y_{t t}(x, t)+y_{x x x x}(x, t)=0, \quad 0<x<1, t>0, \\
y(0, t)=y_{x}(0, t)=0, \\
y_{x x}(1, t)=-k_{1} y_{x t}(1, t), \quad k_{1} \geq 0, \\
y_{x x x}(1, t)=k_{2} y_{t}(1, t), \quad k_{2} \geq 0 .
\end{array}\right.
$$

In this paper, we shall develop parallel results for the same system with variable coefficients. What makes it unique compared to the case of constant coefficients is that with variable coefficients both the characteristic equation and the analytic expression of the eigenfunctions have no explicit formulae. The asymptotic technique appears to be essential for the study.

There are two steps usually found in the study of linear systems with variable coefficients. The first is to transform the "dominant term" of the system under study into a uniform "dominant equation" by space scaling and state transformation where no variable coefficient is involved any longer, while the second is to approximate the eigenfunctions of the system by those of the uniform "dominant equation." This

\footnotetext{
* Received by the editors May 19, 2000; accepted for publication (in revised form) September 25, 2001; published electronically March 5, 2002. This research was supported by the National Key Project of China and the National Natural Science Foundation of China.

http://www.siam.org/journals/sicon/40-6/37251.html

${ }^{\dagger}$ Institute of Systems Science, Academy of Mathematics and System Sciences, Academia Sinica, Beijing 100080, China (bzguo@iss03.iss.ac.cn).
} 
fundamental idea comes essentially from Birkhoff's works on asymptotic estimation of the eigenpairs of the linear differential operators with generalized homogeneous boundary conditions done in the beginning of the last century [5]. A comprehensive review can be found in [4]. This approach has been used in dealing with the beam equations with low order perturbation of variable coefficients (see [6], [7], and [8]). A similar adoption can also be found in the study of the string equations with variable coefficients, for which we refer to [9], [10], and [11] as well as the references therein.

By considering a sequence of eigenfunctions rather than whole sequences in the state Hilbert space, the author recently presented a corollary of Bari's theorem on the Riesz basis property [1]. The result greatly simplifies the procedure in establishing the Riesz basis property for systems described by discrete operators in a Hilbert space since the result eliminates the requirement of estimation of low eigenfunctions, which is rather difficult by other methods found in all previous papers [10], [11], [12].

Following the approach used in [1], together with the asymptotic analysis, this paper presents the Riesz basis property for the Euler-Bernoulli beam equation with variable coefficients. Other major contributions include the exponential stability and asymptotic behavior of the systems under boundary feedback control.

In the next section, we shall present the main results of the paper. The proof of the results and some remarks are given in section 3 .

2. Main results. Consider the following nonuniform Euler-Bernoulli beam equation with linear boundary feedback control:

$$
\left\{\begin{array}{l}
\rho(x) y_{t t}(x, t)+\left(E I(x) y_{x x}(x, t)\right)_{x x}=0, \quad 0<x<1, t>0, \\
y(0, t)=y_{x}(0, t)=y_{x x}(1, t)=0, \\
\left(E I(x) y_{x x}\right)_{x}(1, t)=k y_{t}(1, t),
\end{array}\right.
$$

where $x$ stands for the position and $t$ the time. $E I(x)$ is the flexural rigidity of the beam, and $\rho(x)$ is the mass density at $x . k \geq 0$ is a constant feedback gain. Unlike system (1), here we impose only one end feedback control for simplicity of computation because, from Theorem 2.5 below, it is sufficient for the exponential stabilization of the system. Moreover, it does not make much difference from the methodology point of view. Actually, the analysis in this paper can be used to similarly treat the boundary conditions of (1) along the same lines as the analysis in [1].

The total energy of system (2) is

$$
E(t)=\frac{1}{2} \int_{0}^{1}\left[\rho(x) y_{t}^{2}(x, t)+E I(x) y_{x x}^{2}(x, t)\right] d x .
$$

Formally,

$$
\frac{d E(t)}{d t}=-k y_{t}^{2}(x, t) \leq 0
$$

That is, system (2) is dissipative. Throughout this paper, we always assume that

$$
\rho(x), E I(x) \in C^{4}[0,1], E I, \quad \rho>0 .
$$

System (2) will be considered in the energy Hilbert space $\mathbf{H}=H_{E}^{2}(0,1) \times L^{2}(0,1)$, $H_{E}^{2}(0,1)=\left\{f \in H^{2}(0,1) \mid f(0)=f^{\prime}(0)=0\right\}$, in which the inner product induced norm is defined by

$$
\|(f, g)\|_{\mathbf{H}}^{2}=\int_{0}^{1}\left[\rho(x)|g(x)|^{2}+E I(x)\left|f^{\prime \prime}(x)\right|^{2}\right] d x \quad \forall(f, g) \in \mathbf{H} .
$$


Define operator $\mathcal{A}: D(\mathcal{A})(\subset \mathbf{H}) \rightarrow \mathbf{H}$ as

$$
\left\{\begin{array}{l}
\mathcal{A}(f, g)=\left(g,-\frac{1}{\rho(x)}\left(E I(x) f^{\prime \prime}(x)\right)^{\prime \prime}\right), \\
D(\mathcal{A})=\left\{(f, g) \in\left(H_{E}^{2} \cap H^{4}\right) \times H_{E}^{2} \mid f^{\prime \prime}(1)=0,\left(E I f^{\prime \prime}\right)^{\prime}(1)=k g(1)\right\} .
\end{array}\right.
$$

With the operator $\mathcal{A}$ at hand, we can write (2) into an evolutionary equation in $\mathbf{H}$ :

$$
\frac{d}{d t} Y(t)=\mathcal{A} Y(t), Y(t)=\left(y(\cdot, t), y_{t}(\cdot, t)\right) .
$$

When we talk about system (2) later, we mean its abstract formulation (6). We are concerned with the Riesz basis property of (6) in $\mathbf{H}$; that is, we want to know whether the generalized eigenfunctions of $\mathcal{A}$ form an unconditional basis for $\mathbf{H}$. To do this, we need the following spectral property of $\mathcal{A}$.

Lemma 2.1. Let $\mathcal{A}$ be defined by (5). Then $\mathcal{A}^{-1}$ exists and is compact on $\mathbf{H}$. Hence $\sigma(\mathcal{A})$, the spectrum of $\mathcal{A}$, consists only of isolated eigenvalues, which distribute in conjugate pairs on the complex plane. Moreover, the eigenfunction corresponding to $\lambda \in \sigma(\mathcal{A})$ is of the form $\left(\lambda^{-1} \phi, \phi\right)$, where $\phi \neq 0$ satisfies

$$
\left\{\begin{array}{l}
\lambda^{2} \rho(x) \phi(x)+\left(E I(x) \phi^{\prime \prime}(x)\right)^{\prime \prime}=0, \quad 0<x<1, \\
\phi(0)=\phi^{\prime}(0)=\phi^{\prime \prime}(1)=0 \\
\left(E I(x) \phi^{\prime \prime}\right)^{\prime}(1)=\lambda k \phi(1) .
\end{array}\right.
$$

To verify the basis property, we need the asymptotic properties of both eigenvalues and eigenfunctions, which are stated as the following propositions.

Proposition 2.2. Let $\mathcal{A}$ be defined by (5). Then the eigenvalues $\left\{\lambda_{n}, \overline{\lambda_{n}}\right\}$ of $\mathcal{A}$ have the following asymptotic property:

$$
\lambda_{n}=\frac{\rho_{n}^{2}}{h^{2}}, h=\int_{0}^{1}\left(\frac{\rho(\tau)}{E I(\tau)}\right)^{1 / 4} d \tau, \rho_{n}=\frac{1}{\sqrt{2}}\left(n+\frac{1}{2}\right) \pi(1+i)+\mathcal{O}\left(n^{-1}\right) \text { as } n \rightarrow \infty,
$$

where $n$ is a large positive integer and $\overline{\lambda_{n}}$ denotes the complex conjugate of $\lambda_{n}$. Moreover, $\lambda_{n}$ is geometrically simple when $n$ is large enough.

Proposition 2.3. Let $\lambda_{n}$ be defined as in Proposition 2.2. Then there is a solution $\phi_{n}$ to (7) corresponding to $\lambda_{n}$ having the following asymptotic expansion:

$$
\begin{gathered}
-\frac{\sqrt{2}}{4}(1+i) e^{\frac{1}{4} \int_{0}^{z} a(\tau) d \tau} \phi_{n}(x)=\sin (n+\pi / 2) z-\cos (n+\pi / 2) z \\
+e^{-(n+1 / 2) \pi z}+(-1)^{n} e^{-(n+1 / 2) \pi(1-z)}+\mathcal{O}\left(n^{-1}\right), \\
-\frac{\sqrt{2}}{4}(1+i) e^{\frac{1}{4} \int_{0}^{z} a(\tau) d \tau} \lambda_{n}^{-1} \phi_{n}^{\prime \prime}(x)=i\left(\frac{\rho(x)}{E I(x)}\right)^{1 / 2}[\cos (n+\pi / 2) z-\sin (n+\pi / 2) z \\
\left.+e^{-(n+1 / 2) \pi z}+(-1)^{n} e^{-(n+1 / 2) \pi(1-z)}\right]+\mathcal{O}\left(n^{-1}\right),
\end{gathered}
$$

where $z=z(x)$ and $a(z)$ are defined by

$$
\left\{\begin{array}{l}
z=z(x)=\frac{1}{h} \int_{0}^{x}\left(\frac{\rho(\tau)}{E I(\tau)}\right)^{1 / 4} d \tau, h=\int_{0}^{1}\left(\frac{\rho(\tau)}{E I(\tau)}\right)^{1 / 4} d \tau \\
a(z)=\frac{3 h}{2}\left(\frac{\rho(x)}{E I(x)}\right)^{-5 / 4} \frac{d}{d x}\left(\frac{\rho(x)}{E I(x)}\right)+h \frac{2 E I^{\prime}(x)}{E I(x)}\left(\frac{\rho(x)}{E I(x)}\right)^{-1 / 4}
\end{array}\right.
$$


The main result is the following basis property for system (2).

TheOrem 2.4. Let $\mathcal{A}$ be defined by (5). Then the following hold.

(i) There is a sequence of generalized eigenfunctions of $\mathcal{A}$ which forms a Riesz basis for the state space $\mathbf{H}$.

(ii) The eigenvalues $\left\{\lambda_{n}, \overline{\lambda_{n}}\right\}$ of $\mathcal{A}$ have the asymptotic expansion (8).

(iii) All $\lambda \in \sigma(\mathcal{A})$ with sufficiently large modulus are algebraically simple.

Therefore, $\mathcal{A}$ generates a $C_{0}$-group, and, for the semigroup $e^{\mathcal{A t}}$ generated by $\mathcal{A}$, the spectrum-determined growth condition holds: $\omega(\mathcal{A})=S(\mathcal{A})$, where $\omega(\mathcal{A})=$ $\lim _{t \rightarrow \infty} \frac{1}{t}\left\|e^{\mathcal{A} t}\right\|$ is the growth order of $e^{\mathcal{A} t}$ and $S(\mathcal{A})=\sup \{\operatorname{Re} \lambda \mid \lambda \in \sigma(\mathcal{A})\}$ is the spectral bound of $\mathcal{A}$.

Remark 1. From Theorem 2.4 (iii), (9) and (10) are asymptotic expansions for all generalized eigenfunctions of $\mathcal{A}$.

Theorem 2.4 is the fundamental property of system (2). Many other important properties of system (2) can be concluded from Theorem 2.4. The exponential stability stated below is one such important property that has been studied extensively in the past two decades.

THEOREM 2.5. System (2) is exponentially stable for any $k>0$. That is, there are constants $M, \omega>0$ such that the energy $E(t)$ of system (2) satisfies

$$
E(t)=\frac{1}{2} \int_{0}^{1}\left[\rho(x) y_{t}^{2}(x, t)+E I(x) y_{x x}^{2}(x, t)\right] d x \leq M e^{-\omega t} E(0) \quad \forall t \geq 0,
$$

for any initial condition $\left(y(x, 0), y_{t}(x, 0)\right) \in \mathbf{H}$.

Theorem 2.4 will also be applied to the following beam equation with variable viscous damping:

$$
\left\{\begin{array}{l}
\rho(x) y_{t t}(x, t)+b(x) y_{t}(x, t)+\left(E I(x) y_{x x}(x, t)\right)_{x x}=0, \quad 0<x<1, t>0 \\
y(0, t)=y_{x}(0, t)=y_{x x}(1, t)=0 \\
\left(E I(x) y_{x x}\right)_{x}(1, t)=k y_{t}(1, t)
\end{array}\right.
$$

The uniform case of $E I=\rho=$ const, $b \in C[0,1], k=0$ was discussed in [8]. System (12) can be written as

$$
\frac{d}{d t} Y(t)=(\mathcal{A}+\mathcal{B}) Y(t), Y(t)=\left(y(\cdot, t), y_{t}(\cdot, t)\right),
$$

where $\mathcal{A}$ is defined by (5) and $\mathcal{B}$ is a linear bounded operator on $\mathbf{H}$ :

$$
\mathcal{B}(f, g)=(0,-b \cdot g) .
$$

Equation (13) can be put into the generic framework of discrete-type operators perturbed by the linear bounded operator in the Hilbert spaces. First, we introduce the following definition.

Definition 2.6. A linear operator $A$ in a Hilbert space $H$ is called discrete-type, or $[D]$-class for short, if there are Riesz basis $\left\{\phi_{n}\right\}_{1}^{\infty}$ of $H$, complex series $\left\{\lambda_{n}\right\}_{1}^{\infty}$, and an integer $N>0$ such that

(i) $\lim _{n \rightarrow \infty}\left|\lambda_{n}\right|=\infty, \lambda_{n} \neq \lambda_{m}$ as $n, m>N$;

(ii) $A \phi_{n}=\lambda_{n} \phi_{n}, n>N$;

(iii) $A\left[\phi_{1}, \phi_{2}, \ldots, \phi_{N}\right] \subset\left[\phi_{1}, \phi_{2}, \ldots, \phi_{N}\right]$, and $A$ has spectrum $\left\{\lambda_{i}\right\}_{1}^{N}$ in $\left[\phi_{1}, \phi_{2}, \ldots, \phi_{N}\right]$, where $\left[\phi_{1}, \phi_{2}, \ldots, \phi_{N}\right]$ is the linear subspace spanned by $\left\{\phi_{i}\right\}_{1}^{N}$.

Remark 2. Theorem 2.4 shows that $\mathcal{A}$ is of $[D]$-class. 
It is known that any [D]-class operator $A$ must be a discrete operator [14], and for the $C_{0}$-semigroup $e^{A t}$ generated by $A$, the spectrum-determined growth condition holds. The following basic result can be concluded from the proof of a more general result in [14] (see also [15], [16, section V.4]). A short proof will be given in the next section.

Theorem 2.7. Suppose that $A$ is of $[D]$-class satisfying conditions of Definition 2.6 in a Hilbert space $H$. Let $d_{n}=\min _{n \neq m}\left|\lambda_{n}-\lambda_{m}\right|$. If

$$
\sum_{n>N}^{\infty} d_{n}^{-2}<\infty
$$

then, for any linear bounded operator $B$ on $H$, there are constants $C, L>0$, an integer $M>0$, and eigenpairs $\left\{\mu_{n}, \psi_{n}\right\}_{M}^{\infty}$ of $A+B$ such that

(i) $\left|\mu_{n}-\lambda_{n}\right| \leq C$ for all $n \geq M$.

(ii) $\left\|\psi_{n}-\phi_{n}\right\| \leq L d_{n}^{-1}, n \geq M$. Hence $\sum_{n=M}^{\infty}\left\|\psi_{n}-\phi_{n}\right\|^{2}<\infty$.

We can now consider (12). By Remark $1, \mathcal{A}$ is of $[D]$-class. And the spectral separation of $\mathcal{A}$ satisfies $d_{n}^{-1}=\mathcal{O}\left(n^{-1}\right)$. In Remark 4 of the next section, we shall show that $d_{n}$ is never vanishing. Hence Theorem 2.7 can be applied to $(A, B)=(\mathcal{A}, \mathcal{B})$ to get the following parallel result of Theorem 2.4 for system (12).

TheOrem 2.8. Suppose $E I, \rho \in C^{4}[0,1], E I, \rho>0, b \in C[0,1]$. Then the following hold.

(i) $\mathcal{A}+\mathcal{B}$ is of $[D]$-class.

(ii) The eigenvalues $\left\{\mu_{n}, \overline{\mu_{n}}\right\}$ of $\mathcal{A}+\mathcal{B}$ have the asymptotic expansion

$$
\mu_{n}=\lambda_{n}+\mathcal{O}(1) \text { as } n \rightarrow \infty,
$$

where $\lambda_{n}$ is defined by (8).

(iii) The corresponding eigenfunctions $\left\{\left(\mu_{n}^{-1} \psi_{n}, \psi_{n}\right)\right\} \cup\{$ their conjugates $\}$ of $\mathcal{A}+$ $\mathcal{B}$ have the asymptotic expansion

$$
\left(\mu_{n}^{-1} \psi_{n}, \psi_{n}\right)=\left(\lambda_{n}^{-1} \phi_{n}, \phi_{n}\right)+\epsilon_{n} \text { as } n \rightarrow \infty,
$$

where $\phi_{n}$ is defined by (9) and

$$
\left\|\epsilon_{n}\right\|_{\mathbf{H}}=\mathcal{O}\left(n^{-1}\right) .
$$

The following result can be viewed as a consequence of Theorem 2.8.

Corollary 2.9. Let $\left\{\mu_{n}\right\}$ be the eigenvalues of $\mathcal{A}+\mathcal{B}$ determined in Theorem 2.8 . Then

$$
\lim _{n \rightarrow \infty} \operatorname{Re} \mu_{n}=-\frac{1}{2} \frac{\int_{0}^{1} b(x) e^{-\frac{1}{2} \int_{0}^{z} a(\tau) d \tau} d x+4 k e^{-\frac{1}{2} \int_{0}^{1} a(\tau) d \tau}}{\int_{0}^{1} \rho(x) e^{-\frac{1}{2} \int_{0}^{z} a(\tau) d \tau} d x},
$$

where $z=z(x), a(z)$ are defined in (11).

Corollary 2.9 concludes some existing results for system (12). We give several examples below.

Example 1. Suppose that $\rho=1, k=0$, and $E I$ is a constant. Then $a=0$. Equation (19) becomes

$$
\lim _{n \rightarrow \infty} \operatorname{Re} \mu_{n}=-\frac{1}{2} \int_{0}^{1} b(x) d x
$$


which is a strengthened conjecture

$$
\lim _{n \rightarrow \infty} \frac{\sum_{j \leq n} \operatorname{Re} \mu_{j}}{n}=-\frac{1}{2} \int_{0}^{1} b(x) d x
$$

made in [13] for the same system with hinged boundary conditions and resolved later in [6] under the assumption that $b(x) \geq 0$. However, we do not impose any assumption on the symbol of $b$.

Example 2. Suppose that $\rho=E I=1, k=0$. Then (12) becomes

$$
\left\{\begin{array}{l}
y_{t t}(x, t)+b(x) y_{t}(x, t)+y_{x x x x}(x, t)=0, \quad 0<x<1, t>0 \\
y(0, t)=y_{x}(0, t)=y_{x x}(1, t)=y_{x x x}(1, t)=0
\end{array}\right.
$$

which is just the system studied in [8]. Equation (20) holds for this system. However, our result shows that the main hypothesis (1.3) of [8] is nothing but $\int_{0}^{1} b(x) d x>0$. Moreover, from our discussion, Theorem 2.8 is sufficient to derive (20). This is because spectral analysis for system (22) with $b=0$ is quite simple and does not necessarily need to rely on Theorem 2.4 [1].

Example 3. Suppose that $b(x) \geq 0$ for $x \in[0,1]$, and $b(x)>b_{0}>0$ for all $x$ in some subset $(a, b) \subset[0,1]$ in $(22)$. The system is then exponentially stable. When $k=0, b(x) \geq 0$ for $x \in[0,1]$ and $b(x)>b_{0}>0$ for all $x$ in some subset $(a, b) \subset[0,1]$, system (12) is also exponentially stable. However, the method used in [18] appears to be unavailable for this case. We will give a short interpretation for Example 3 in section 3 .

Finally, we present a high order approximation of the eigenvalues of system (12).

Proposition 2.10. Suppose (3) and

$$
b(x) \in C^{1}[0,1], \quad \int_{0}^{1} b(x) e^{-\frac{1}{2} \int_{0}^{z} a(\tau) d \tau} d x+4 k e^{-\frac{1}{2} \int_{0}^{1} a(\tau) d \tau}>0 .
$$

Then the eigenvalues $\left\{\mu_{n}, \overline{\mu_{n}}\right\}$ of $\mathcal{A}+\mathcal{B}$ have the asymptotic expansion

(24) $\mu_{n}=-\frac{2 \tilde{k}}{h^{2}}+i\left[(n+1 / 2) \frac{\pi}{h}\right]^{2}-\frac{i}{2 h^{2}} \int_{0}^{1} a_{1}(\tau) d \tau-\frac{1}{2 h^{2}} \int_{0}^{1} \tilde{b}(\tau) d \tau+\mathcal{O}\left(n^{-1}\right)$,

where $\tilde{b}(z), a_{1}(z)$, and $\tilde{k}$ are given by

$$
\begin{gathered}
\tilde{k}=\frac{k h}{E I(1)}\left(\frac{\rho(1)}{E I(1)}\right)^{-3 / 4}, \\
\tilde{b}(z)=\frac{b(x)}{\rho(x)}, z=\frac{1}{h} \int_{0}^{x}\left(\frac{\rho(\tau)}{E I(\tau)}\right)^{1 / 4} d \tau, \\
a_{1}(z)=-\frac{3}{2} a^{\prime}(z)-\frac{9}{16} a^{2}(z)-\frac{1}{4} a(z) .
\end{gathered}
$$

3. Proof of main results.

Proof of Lemma 2.1. A direct calculation shows that

$$
\mathcal{A}^{-1}(f, g)=(\phi, \psi) \text { for any }(f, g) \in \mathrm{H},
$$


where

$$
\begin{cases}\psi & =f, \\ \phi(x) & =k f(1) \int_{0}^{x}(x-\tau) \frac{\tau-1}{E I(\tau)} d \tau+\int_{0}^{x} \rho(\tau) g(\tau) d \tau \int_{\tau}^{x} d \vartheta \int_{\tau}^{\vartheta} \frac{s-\tau}{E I(s)} d s .\end{cases}
$$

The compactness follows from the Sobolev embedding theorem. Other conclusions are obvious, and the details are omitted.

In order to study the asymptotic behavior of the solution of (7), we rewrite (7) in a standard form of the eigenproblem of a linear differential operator with generalized homogeneous boundary conditions:

$$
\left\{\begin{array}{l}
\phi^{(4)}(x)+\frac{2 E I^{\prime}(x)}{E I(x)} \phi^{\prime \prime \prime}(x)+\frac{E I^{\prime \prime}(x)}{E I(x)} \phi^{\prime \prime}(x)+\lambda^{2} \frac{\rho(x)}{E I(x)} \phi(x)=0, \\
\phi(0)=\phi^{\prime}(0)=\phi^{\prime \prime}(1)=0, \\
\phi^{\prime \prime \prime}(1)=\lambda \frac{k}{E I(1)} \phi(1) .
\end{array}\right.
$$

Two basic transformations are essential. First, the "dominant term," $\phi^{(4)}(x)+$ $\lambda^{2} \rho(x) / E I(x) \phi(x)$ of (28), is transformed to become a uniform form by space scaling. In fact, set

$$
\phi(x)=f(z), z=z(x)=\frac{1}{h} \int_{0}^{x}\left(\frac{\rho(\tau)}{E I(\tau)}\right)^{1 / 4} d \tau, h=\int_{0}^{1}\left(\frac{\rho(\tau)}{E I(\tau)}\right)^{1 / 4} d \tau .
$$

Then $f$ satisfies

$$
\left\{\begin{array}{l}
f^{(4)}(z)+a(z) f^{\prime \prime \prime}(z)+b_{f}(z) f^{\prime \prime}(z)+c(z) f^{\prime}(z)+\lambda^{2} h^{4} f(z)=0 \\
f(0)=f^{\prime}(0)=0 \\
f^{\prime \prime}(1)+a_{0} f^{\prime}(1)=0 \\
f^{\prime \prime \prime}(1)=b_{0} f^{\prime}(1)+\lambda \frac{k h^{3}}{E I(1)}\left(\frac{\rho(1)}{E I(1)}\right)^{-3 / 4} f(1)
\end{array}\right.
$$

where $a_{0}$ and $b_{0}$ are constants depending on $h, \rho^{(i)}(1), E I^{(i)}(1), i=0,1,2, b_{f}(z)$ and $c(z)$ are the smooth functions of $h, \rho^{(i)}(x), E I^{(i)}(x), i=0,1,2,3$ through $z=z(x)$ defined by (11), and $a(z)$ is the function given by (11).

Second, in order to cancel the term $a(z) f^{\prime \prime \prime}$ in (30) as was done in [4], we make the invertible state transformation

$$
f(z)=e^{-\frac{1}{4} \int_{0}^{z} a(\tau) d \tau} g(z) .
$$

Then $g$ satisfies

$$
\left\{\begin{array}{l}
g^{(4)}(z)+a_{1}(z) g^{\prime \prime}(z)+a_{2}(z) g^{\prime}(z)+a_{3}(z) g(z)+\lambda^{2} h^{4} g(z)=0 \\
g(0)=g^{\prime}(0)=0 \\
g^{\prime \prime}(1)=a_{11} g^{\prime}(1)+a_{12} g(1) \\
g^{\prime \prime \prime}(1)=a_{21} g^{\prime}(1)+\left[\lambda \frac{k h^{3}}{E I(1)}\left(\frac{\rho(1)}{E I(1)}\right)^{-3 / 4}+a_{22}\right] g(1),
\end{array}\right.
$$

where $a_{i j}, i, j=1,2$ are some real constants depending on $h, \rho^{(i)}(1), E I^{(i)}(1), i=$ $0,1,2, a_{2}(z)$ and $a_{3}(z)$ are the smooth functions of $h, \rho^{(i)}(x), E I^{(i)}(x), i=0,1,2,3$ through $z=z(x)$ defined by $(29)$, and $a_{1}(z)$ is given by $(27)$. 
It can be seen that (7) and (32) are equivalent. Our next task is to use the eigenpairs of the uniform "dominant term," $g^{(4)}(z)+\lambda^{2} h^{4} g(z)=0$ of (32), to approximate those of the whole system. Note that when $k=0,(32)$ is in the standard form of a linear differential operator with generalized homogeneous boundary conditions, which was studied in [4] in greater detail.

Now we proceed as in section 4, Chapter 2 of [4] to estimate asymptotically the solutions to (32). Since system (2) is dissipative, all eigenvalues are located on the left half complex plane. Due to the conjugate property of the eigenvalues, we may consider only those $\lambda$ with $\pi / 2 \leq \arg \lambda \leq \pi$.

Let $\lambda=\rho^{2} / h^{2}$. Then, as $\pi / 2 \leq \arg \lambda \leq \pi$,

$$
\pi / 4 \leq \arg \rho \leq \pi / 2 .
$$

Now set

$$
\left\{\begin{array}{l}
\omega_{1}=e^{3 / 4 \pi i}, \omega_{2}=e^{\pi / 4 i}, \omega_{3}=-\omega_{2}, \omega_{4}=-\omega_{1}, \\
S=\left\{\rho \mid \frac{\pi}{4} \leq \arg \rho \leq \frac{\pi}{2}\right\} .
\end{array}\right.
$$

In what follows, $\rho$ is always assumed to be in $S$. Note that

$$
\operatorname{Re}\left(\rho \omega_{1}\right) \leq \operatorname{Re}\left(\rho \omega_{2}\right) \leq \operatorname{Re}\left(\rho \omega_{3}\right) \leq \operatorname{Re}\left(\rho \omega_{4}\right) \quad \forall \rho \in S .
$$

The following important facts are used frequently in what follows.

$$
\left\{\begin{array}{l}
\operatorname{Re}\left(\rho \omega_{1}\right)=-|\rho| \sin \left(\arg \rho+\frac{\pi}{4}\right) \leq-\sqrt{2} / 2|\rho|<0, \\
\operatorname{Re}\left(\rho \omega_{2}\right)=|\rho| \cos \left(\arg \rho+\frac{\pi}{4}\right) \leq 0 .
\end{array}\right.
$$

Lemma 3.1 comes from Theorem 2.4 in section 4, Chapter 2 of [4].

Lemma 3.1. For $|\rho|$ large enough, $\rho \in S$, there are four linearly independent solutions $g_{k}(z), k=1,2,3,4$ to

$$
g^{(4)}(z)+a_{1}(z) g^{\prime \prime}(z)+a_{2}(z) g^{\prime}(z)+a_{3}(z) g(z)+\rho^{4} g(z)=0,
$$

such that

$$
\left\{\begin{array}{l}
g_{k}(z)=e^{\rho \omega_{k} z}\left[1+\mathcal{O}\left(\frac{1}{\rho}\right)\right] \\
g_{k}^{\prime}(z)=\rho \omega_{k} e^{\rho \omega_{k} z}\left[1+\mathcal{O}\left(\frac{1}{\rho}\right)\right] \\
g_{k}^{\prime \prime}(z)=\left(\rho \omega_{k}\right)^{2} e^{\rho \omega_{k} z}\left[1+\mathcal{O}\left(\frac{1}{\rho}\right)\right] \\
g_{k}^{\prime \prime \prime}(z)=\left(\rho \omega_{k}\right)^{3} e^{\rho \omega_{k} z}\left[1+\mathcal{O}\left(\frac{1}{\rho}\right)\right]
\end{array}\right.
$$

With these preparations, we come to the proof of Proposition 2.2.

Proof of Proposition 2.2. Let $g(z)$ be a solution of (32). There are constants $c_{i}, i=1,2,3,4$, such that

$$
g(z)=c_{1} g_{1}(z)+c_{2} g_{2}(z)+c_{3} g_{3}(z)+c_{4} g_{4}(z),
$$

where $g_{k}(z), k=1,2,3,4$ are defined by (37). By boundary conditions, $c_{i}, i=1,2,3,4$ are solutions to the following system of linear algebraic equations:

$$
\left\{\begin{array}{l}
c_{1} g_{1}(0)+c_{2} g_{2}(0)+c_{3} g_{3}(0)+c_{4} g_{4}(0)=0, \\
c_{1} g_{1}^{\prime}(0)+c_{2} g_{2}^{\prime}(0)+c_{3} g_{3}^{\prime}(0)+c_{4} g_{4}^{\prime}(0)=0, \\
{\left[g_{1}^{\prime \prime}(1)-a_{11} g_{1}^{\prime}(1)-a_{12} g_{1}(1)\right] c_{1}+\left[g_{2}^{\prime \prime}(1)-a_{11} g_{2}^{\prime}(1)-a_{12} g_{2}(1)\right] c_{2}} \\
\quad\left[g^{\prime \prime}(1)-a_{11} g_{3}^{\prime}(1)-a_{12} g_{3}(1)\right] c_{3}+\left[g_{4}^{\prime \prime}(1)-a_{11} g_{4}^{\prime}(1)-a_{12} g_{4}(1)\right] c_{4}=0, \\
{\left[g_{1}^{\prime \prime \prime}(1)-a_{21} g_{1}^{\prime}(1)-a_{22} g_{1}(1)-\tilde{k} \rho^{2} g_{1}(1)\right] c_{1}+\left[g_{2}^{\prime \prime \prime}(1)-a_{21} g_{2}^{\prime}(1)-a_{22} g_{2}(1)-\tilde{k} \rho^{2} g_{2}(1)\right] c_{2}} \\
\quad+\left[g_{3}^{\prime \prime \prime}(1)-a_{21} g_{3}^{\prime}(1)-a_{22} g_{3}(1)-\tilde{k} \rho^{2} g_{3}(1)\right] c_{3}+\left[g_{4}^{\prime \prime \prime}(1)-a_{21} g_{4}^{\prime}(1)-a_{22} g_{4}(1)-\tilde{k} \rho^{2} g_{4}(1)\right] c_{4}=0,
\end{array}\right.
$$


where $\tilde{k}$ is defined by $(25)$.

From (36) and (37), for any $k, 1 \leq k \leq 4$,

$$
\left\{\begin{array}{l}
g_{k}(0)=1+\mathcal{O}\left(\frac{1}{\rho}\right), g_{k}^{\prime}(0)=\rho \omega_{k}\left[1+\mathcal{O}\left(\frac{1}{\rho}\right)\right] \\
{\left[g_{k}^{\prime \prime}(1)-a_{11} g_{k}^{\prime}(1)-a_{12} g_{k}(1)\right]=\left(\rho \omega_{k}\right)^{2} e^{\rho \omega_{k}}\left[1+\mathcal{O}\left(\frac{1}{\rho}\right)\right]} \\
g_{k}^{\prime \prime \prime}(1)-a_{21} g_{k}^{\prime}(1)-a_{22} g_{k}(1)-\tilde{k} \rho^{2} g_{k}(1) \\
=\left(\rho \omega_{k}\right)^{3} e^{\rho \omega_{k}}\left[1+\mathcal{O}\left(\frac{1}{\rho}\right)\right]-\tilde{k} \rho^{2} e^{\rho \omega_{k}}\left[1+\mathcal{O}\left(\frac{1}{\rho}\right)\right]
\end{array}\right.
$$

and

$$
\left|e^{\rho \omega_{2}}\right| \leq 1,\left|e^{\rho \omega_{1}}\right|=\mathcal{O}\left(e^{-c|\rho|}\right) \text { as }|\rho| \rightarrow \infty,
$$

for some constant $c>0$. Then we know that $g(z)$ is nonzero if and only if $\rho$ satisfies the characteristic equation

$$
\operatorname{det}\left(\begin{array}{cccc}
{[1]} & {[1]} & {[1]} & {[1]} \\
\rho \omega_{1}[1] & \rho \omega_{2}[1] & \rho \omega_{3}[1] & \rho \omega_{4}[1] \\
\left(\rho \omega_{1}\right)^{2} e^{\rho \omega_{1}}[1] & \left(\rho \omega_{2}\right)^{2} e^{\rho \omega_{2}}[1] & \left(\rho \omega_{3}\right)^{2} e^{\rho \omega_{3}}[1] & \left(\rho \omega_{4}\right)^{2} e^{\rho \omega_{4}}[1] \\
\left(\rho \omega_{1}\right)^{3} e^{\rho \omega_{1}}[1] & \left(\rho \omega_{2}\right)^{3} e^{\rho \omega_{2}}[1] & \left(\rho \omega_{3}\right)^{3} e^{\rho \omega_{3}[1]} & \left(\rho \omega_{4}\right)^{3} e^{\rho \omega_{4}[1]}
\end{array}\right)=0,
$$

where $[1]=1+\mathcal{O}\left(\frac{1}{\rho}\right)$. Since $\omega_{4}=-\omega_{1}, \omega_{3}=-\omega_{2}$, the above equation is equivalent to

$$
\operatorname{det}\left(\begin{array}{cccc}
{[1]} & {[1]} & e^{\rho \omega_{2}[1]} & e^{\rho \omega_{1}}[1] \\
\omega_{1}[1] & \omega_{2}[1] & -\omega_{2} e^{\rho \omega_{2}}[1] & -\omega_{1} e^{\rho \omega_{1}}[1] \\
\omega_{1}^{2} e^{\rho \omega_{1}}[1] & \omega_{2}^{2} e^{\rho \omega_{2}}[1] & \omega_{2}^{2}[1] & \omega_{1}^{2}[1] \\
\omega_{1}^{3} e^{\rho \omega_{1}}[1] & \omega_{2}^{3} e^{\rho \omega_{2}}[1] & -\omega_{2}^{3}[1] & -\omega_{1}^{3}[1]
\end{array}\right)=0 .
$$

Noting that each element of the matrix in (42) is bounded, we may rewrite (42) as

$$
\operatorname{det}\left(\begin{array}{cccc}
1 & 1 & e^{\rho \omega_{2}} & 0 \\
\omega_{1} & \omega_{2} & -\omega_{2} e^{\rho \omega_{2}} & 0 \\
0 & \omega_{2}^{2} e^{\rho \omega_{2}} & \omega_{2}^{2} & \omega_{1}^{2} \\
0 & \omega_{2}^{3} e^{\rho \omega_{2}} & -\omega_{2}^{3} & -\omega_{1}^{3}
\end{array}\right)+\mathcal{O}\left(\frac{1}{\rho}\right)=0
$$

which results in

$$
e^{2 \rho \omega_{2}}=\left(\frac{\omega_{2}-\omega_{1}}{\omega_{2}+\omega_{1}}\right)^{2}+\mathcal{O}\left(\frac{1}{\rho}\right)=-1+\mathcal{O}\left(\frac{1}{\rho}\right) .
$$

By solving (44), we obtain (8) by the same arguments as those of section 4 , Chapter 2 of [4]. Since the matrix in (43) has rank 3 for each sufficiently large $\rho_{n}$, there is only one linearly independent solution $g$ to (32) for $\rho=\rho_{n}$. Hence each $\lambda_{n}$ is geometrically simple for $n$ sufficiently large.

In Remark 4, we will indicate that each eigenvalue of $\mathcal{A}$ must be geometrically simple. Noting (37), (38), and (42), we can write $g, g^{\prime \prime}$ as

$$
g(z)=\operatorname{det}\left(\begin{array}{cccc}
{[1]} & {[1]} & e^{\rho \omega_{2}}[1] & e^{\rho \omega_{1}}[1] \\
e^{\rho \omega_{1} z}[1] & e^{\rho \omega_{2} z}[1] & e^{\rho \omega_{2}(1-z)}[1] & e^{\rho \omega_{1}(1-z)}[1] \\
\omega_{1}^{2} e^{\rho \omega_{1}}[1] & \omega_{2}^{2} e^{\rho \omega_{2}}[1] & \omega_{2}^{2}[1] & \omega_{1}^{2}[1] \\
\omega_{1}^{3} e^{\rho \omega_{1}}[1] & \omega_{2}^{3} e^{\rho \omega_{2}}[1] & -\omega_{2}^{3}[1] & -\omega_{1}^{3}[1]
\end{array}\right)
$$

(46) $g^{\prime \prime}(z)=\rho^{2} \operatorname{det}\left(\begin{array}{cccc}\omega_{1}^{2} e^{\rho \omega_{1} z}[1] & \omega_{2}^{2} e^{\rho \omega_{2} z}[1] & \omega_{2}^{2} e^{\rho \omega_{2}[1]} & e^{\rho \omega_{1}[1]} \\ \omega_{1}^{2} e^{\rho \omega_{1}}[1] & \omega_{2}^{2} e^{\rho \omega_{2}}[1] & \omega_{2}^{2}[1] & \omega_{1}^{2} e^{\rho \omega_{1}(1-z)}[1] \\ \omega_{1}^{3} e^{\rho \omega_{1}}[1] & \omega_{2}^{3} e^{\rho \omega_{2}[1]} & -\omega_{2}^{3}[1] & \omega_{1}^{2}[1] \\ -\omega_{1}^{3}[1]\end{array}\right)$. 
Lemma 3.2. Let $\lambda_{n}, \rho_{n}$ be defined as in Proposition 2.2. Then the unique (up to a scalar) associated solution $g_{n}$ to (32) has the following asymptotic expansion:

$$
\begin{aligned}
-\frac{\sqrt{2}}{4}(1+i) g_{n}(z)= & \sin (n+\pi / 2) z-\cos (n+\pi / 2) z+e^{-(n+1 / 2) \pi z} \\
& +(-1)^{n} e^{-(n+1 / 2) \pi(1-z)}+\mathcal{O}\left(n^{-1}\right),
\end{aligned}
$$

$$
\begin{aligned}
-\frac{\sqrt{2}}{4}(1+i) \rho_{n}^{-2} g_{n}^{\prime \prime}(z)= & i[\cos (n+\pi / 2) z-\sin (n+\pi / 2) z \\
& \left.+e^{-(n+1 / 2) \pi z}+(-1)^{n} e^{-(n+1 / 2) \pi(1-z)}\right]+\mathcal{O}\left(n^{-1}\right)
\end{aligned}
$$

Moreover, it follows directly from (37) and (45) that

$$
\rho_{n}^{-2} g_{n}^{\prime}(z)=\mathcal{O}\left(n^{-1}\right) .
$$

Proof. It follows from (45) that

$$
g_{n}(z)=\operatorname{det}\left(\begin{array}{cccc}
1 & 1 & e^{\rho_{n} \omega_{2}} & 0 \\
e^{\rho_{n} \omega_{1} z} & e^{\rho_{n} \omega_{2} z} & e^{\rho_{n} \omega_{2}(1-z)} & e^{\rho_{n} \omega_{1}(1-z)} \\
0 & \omega_{2}^{2} e^{\rho_{n} \omega_{2}} & \omega_{2}^{2} & \omega_{1}^{2} \\
0 & \omega_{2}^{3} e^{\rho_{n} \omega_{2}} & -\omega_{2}^{3} & -\omega_{1}^{3}
\end{array}\right)+\mathcal{O}\left(\frac{1}{\rho_{n}}\right) .
$$

After a simple calculation, we find that

$$
\begin{gathered}
g_{n}(z)=\omega_{1}^{2} \omega_{2}^{2}\left[2 \omega_{1} e^{\rho_{n} \omega_{1} z}+2 \omega_{2} e^{\rho_{n} \omega_{2}} e^{\rho_{n} \omega_{1}(1-z)}\right. \\
\left.\quad+\left(\omega_{2}+\omega_{1}\right) e^{\rho_{n} \omega_{2}} e^{\rho_{n} \omega_{2}(1-z)}+\left(\omega_{2}-\omega_{1}\right) e^{\rho_{n} \omega_{2} z}\right]+\mathcal{O}\left({\frac{1}{\rho_{n}}}_{n}\right) \\
=\sqrt{2}(i-1)[\sin (n+\pi / 2) z-\cos (n+\pi / 2) z \\
\left.+e^{-(n+1 / 2) \pi z}+(-1)^{n} e^{-(n+1 / 2) \pi(1-z)}\right]+\mathcal{O}\left(\frac{1}{n}\right) .
\end{gathered}
$$

This is (47). Equation (48) can be proved similarly.

Note that the asymptotic expansions (47) and (48) are exactly the same as those obtained in [1] for the eigenfunctions of system (2) with constant coefficients; i.e., $E I=\rho=$ const. However, it should be pointed out that the estimates in [1] and [2] rely on the analytic expression of the eigenfunctions.

Proof of Proposition 2.3. The result follows directly from the following facts that are deduced from transformations (29), (31), and (49):

$$
\left\{\begin{array}{l}
-\frac{\sqrt{2}}{4}(1+i) e^{\frac{1}{4} \int_{0}^{z} a(\tau) d \tau} f_{n}(z)=-\frac{\sqrt{2}}{4}(1+i) g_{n}(z) \\
-\frac{\sqrt{2}}{4}(1+i) e^{\frac{1}{4} \int_{0}^{z} a(\tau) d \tau} \rho_{n}^{-2} f_{n}^{\prime \prime}(z)=-\frac{\sqrt{2}}{4}(1+i) \rho_{n}^{-2} g_{n}^{\prime \prime}(z)+\mathcal{O}\left(\frac{1}{n}\right) \\
\phi_{n}(x)=f_{n}(z), \rho_{n}^{-2} \phi_{n}^{\prime \prime}(x)=\frac{1}{h^{2}}\left(\frac{\rho(x)}{E I(x)}\right)^{1 / 2} \rho_{n}^{-2} f_{n}^{\prime \prime}(z)+\mathcal{O}\left(\frac{1}{n}\right)
\end{array}\right.
$$

Before proving Theorem 2.4, let us recall that for a closed linear operator A in a Hilbert space $H$, a nonzero $x \in H$ is called a generalized eigenvector of $\mathrm{A}$, corresponding to an eigenvalue $\lambda$ of $\mathrm{A}$ which has finite algebraic multiplicity, if there is a positive integer $n$ such that $(\lambda-\mathrm{A})^{n} x=0$. A sequence $\left\{x_{n}\right\}_{1}^{\infty}$ in $H$ is called a 
Riesz basis for $H$ if there is an orthonormal basis $\left\{e_{n}\right\}_{1}^{\infty}$ in $H$ and a linear bounded invertible operator $\mathrm{T}$ such that

$$
\mathrm{T} e_{n}=x_{n}, n=1,2, \ldots
$$

It is seen that each Riesz basis sequence must be approximately normalized:

$$
C_{1} \leq\left\|x_{n}\right\| \leq C_{2}, C_{1}, C_{2}>0, n=1,2, \ldots
$$

Suppose that $\left\{\lambda_{n}\right\}_{1}^{\infty} \subset \sigma(\mathrm{A})$ and lie in some left half complex plane. If each $\lambda_{n}$ has finite algebraic multiplicity $m_{n}$ and $m_{n}=1$ as $n>N$ for some integer $N>1$, then there is a sequence of linearly independent generalized eigenvectors $\left\{x_{n_{i}}\right\}_{i=1}^{m_{n}}$ corresponding to $\lambda_{n}$. If $\left\{\left\{x_{n_{i}}\right\}_{i=1}^{m_{n}}\right\}_{n=1}^{\infty}$ forms a Riesz basis for $H$, then A generates a $C_{0}$-semigroup $e^{\mathrm{A} t}$ which can be represented as

$$
e^{\mathrm{A} t} x=\sum_{n=1}^{\infty} e^{\lambda_{n} t} \sum_{i=1}^{m_{n}} a_{n i} \sum_{j=1}^{m_{n}} f_{n j}(t) x_{n_{j}} \text { for any } x=\sum_{n=1}^{\infty} \sum_{i=1}^{m_{n}} a_{n i} x_{n_{i}} \in H,
$$

where $f_{n j}(t)$ is a polynomial of $t$ with order less than $m_{n}$. In particular, if $a<\operatorname{Re} \lambda<b$ for some real numbers $a$ and $b$, then A generates a $C_{0}$-group on $H$. Moreover, the spectrum-determined growth condition holds for $e^{\mathrm{A} t}$.

In order to remove the requirement of the estimation of the low eigenpairs of the system, a corollary of Bari's theorem is recently reported in [1] (a simplified proof can be found in [2]), which provides a much less demanding approach in generating a Riesz basis for general discrete operators in the Hilbert spaces. The result is cited here.

TheOREM 3.3. Let A be a densely defined discrete operator (that is, $(\lambda-\mathrm{A})^{-1}$ is compact for some $\lambda$ ) in a Hilbert space $H$. Let $\left\{z_{n}\right\}_{1}^{\infty}$ be a Riesz basis for $H$. If there are an $N \geq 0$ and a sequence of generalized eigenvectors $\left\{x_{n}\right\}_{N+1}^{\infty}$ of A such that

$$
\sum_{N+1}^{\infty}\left\|x_{n}-z_{n}\right\|^{2}<\infty
$$

then

(i) There are an $M>N$ and generalized eigenvectors $\left\{x_{n 0}\right\}_{1}^{M}$ of $\mathrm{A}$ such that $\left\{x_{n 0}\right\}_{1}^{M} \cup\left\{x_{n}\right\}_{M+1}^{\infty}$ forms a Riesz basis for $H$.

(ii) Consequently, let $\left\{x_{n 0}\right\}_{1}^{M} \cup\left\{x_{n}\right\}_{M+1}^{\infty}$ correspond to eigenvalues $\left\{\sigma_{n}\right\}_{1}^{\infty}$ of $\mathrm{A}$. Then $\sigma(\mathrm{A})=\left\{\sigma_{n}\right\}_{1}^{\infty}$, where $\sigma_{n}$ is counted according to its algebraic multiplicity.

(iii) If there is an $M_{0}>0$ such that $\sigma_{n} \neq \sigma_{m}$ for all $m, n>M_{0}$, then there is an $N_{0}>M_{0}$ such that all $\sigma_{n}, n>N_{0}$ are algebraically simple.

Remark 3. It follows from Theorem 3.3 that when $A$ and $B$ satisfy the conditions (i) and (ii) of Theorem 2.7, $A+B$ is of $[D]$-class.

In order to apply Theorem 3.3 to the operator $\mathcal{A}$ when we consider $\left\{x_{n}\right\}$ in Theorem 3.3 as the eigenfunctions of $\mathcal{A}$, we need a referring Riesz basis $\left\{z_{n}\right\}_{1}^{\infty}$ as well. For the system (2), this is accomplished by collecting (approximately) normalized eigenfunctions of the following free conservative system:

$$
\left\{\begin{array}{l}
\rho(x) y_{t t}(x, t)+\left(E I(x) y_{x x}(x, t)\right)_{x x}=0, \quad 0<x<1, t>0 \\
y(0, t)=y_{x}(0, t)=y_{x x}(1, t)=\left(E I y_{x x}\right)_{x}(1, t)=0 .
\end{array}\right.
$$


The system operator $\mathcal{A}_{0}: D\left(\mathcal{A}_{0}\right)(\subset \mathrm{H}) \rightarrow \mathrm{H}$ associated with (51) is nothing but the operator $\mathcal{A}$ with $k=0$ :

$$
\left\{\begin{array}{l}
\mathcal{A}_{0}(f, g)=\left(g,-\frac{1}{\rho(x)}\left(E I(x) f^{\prime \prime}(x)\right)^{\prime \prime}\right) \\
D\left(\mathcal{A}_{0}\right)=\left\{(f, g) \in\left(H_{E}^{2} \cap H^{4}\right) \times H_{E}^{2} \mid f^{\prime \prime}(0)=f^{\prime \prime \prime}(1)=0\right\}
\end{array}\right.
$$

$\mathcal{A}_{0}$ is skew-adjoint with compact resolvent in H. Since Propositions 2.2 and 2.3 still keep valid when $k=0$, we have the following counterpart for the operator $\mathcal{A}_{0}$.

LEMma 3.4. Each $\mu \in \sigma\left(\mathcal{A}_{0}\right)$, with sufficiently large modulus, is geometrically simple and hence algebraically simple. The eigenvalues $\left\{\lambda_{n 0}, \overline{\lambda_{n 0}}\right\}$ and the corresponding eigenfunctions $\left\{\left(\lambda_{n 0}^{-1} \phi_{n 0}, \phi_{n 0}\right)\right\} \cup\{$ their conjugates $\}$ of $\mathcal{A}_{0}$ have the following asymptotic expressions:

$\lambda_{n 0}=\frac{\rho_{n}^{2}}{h^{2}}, h=\int_{0}^{1}\left(\frac{\rho(\tau)}{E I(\tau)}\right)^{1 / 4} d \tau, \rho_{n}=\frac{1}{\sqrt{2}}\left(n+\frac{1}{2}\right) \pi(1+i)+\mathcal{O}\left(n^{-1}\right)$ as $n \rightarrow \infty$,

where $n$ is a large positive integer, and

$$
\left\{\begin{array}{l}
-\frac{\sqrt{2}}{4}(1+i) e^{\frac{1}{4} \int_{0}^{z} a(\tau) d \tau} \phi_{n 0}(x)=\sin (n+\pi / 2) z-\cos (n+\pi / 2) z \\
+e^{-(n+1 / 2) \pi z}+(-1)^{n} e^{-(n+1 / 2) \pi(1-z)}+\mathcal{O}\left(n^{-1}\right), \\
-\frac{\sqrt{2}}{4}(1+i) e^{\frac{1}{4} \int_{0}^{z} a(\tau) d \tau} \lambda_{n 0}^{-1} \phi_{n 0}^{\prime \prime}(x)=i\left(\frac{\rho(x)}{E I(x)}\right)^{1 / 2}[\cos (n+\pi / 2) z-\sin (n+\pi / 2) z \\
\left.+e^{-(n+1 / 2) \pi z}+(-1)^{n} e^{-(n+1 / 2) \pi(1-z)}\right]+\mathcal{O}\left(n^{-1}\right) .
\end{array}\right.
$$

Proof of Theorem 2.4. Since $\mathcal{A}_{0}$ is a skew-adjoint discrete operator in $\mathrm{H}$, from a well-known result in functional analysis, the set of all $\omega$-linearly independent eigenfunctions of $\mathcal{A}_{0}$ forms an orthogonal basis for $\mathrm{H}$. Since $\left(\phi_{n 0}, \lambda_{n 0} \phi_{n 0}\right)$ defined by (54) are approximately normalized, $\left\{\left(\phi_{n 0}, \lambda_{n 0} \phi_{n 0}\right)\right\} \cup\{$ their conjugates $\}$ form a (orthogonal) Riesz basis for H. Combining (9), (10), (53), and (54), we see that there is an $N>0$ such that

$$
\sum_{n>N}^{\infty}\left\|\left(\lambda_{n}^{-1} \phi_{n}, \phi_{n}\right)-\left(\lambda_{n 0}^{-1} \phi_{n 0}, \phi_{n 0}\right)\right\|_{\mathrm{H}}^{2}=\sum_{n>N}^{\infty} \mathcal{O}\left(n^{-2}\right)<\infty .
$$

The same is true for their conjugates. Hence the conditions of Theorem 2.5 are satisfied with correspondence $\mathcal{A}=A, x_{n}=\left(\lambda_{n}^{-1} \phi_{n}, \phi_{n}\right), z_{n}=\left(\lambda_{n 0}^{-1} \phi_{n 0}, \phi_{n 0}\right)$. The proof is complete.

Now we are in a position to show the exponential stability confirmed by Theorem 2.7. Since the spectrum-determined growth condition holds, which is claimed by Theorem 2.4, system (2) is exponentially stable if and only if there is an $\omega>0$ such that

$$
\operatorname{Re} \lambda<-\omega \quad \forall \lambda \in \sigma(\mathcal{A}) .
$$

Lemma 3.5. Let $\lambda_{n}$ be defined by (8). Then there is an $\omega_{0}>0$ such that

$$
\lim _{n \rightarrow \infty} \operatorname{Re} \lambda_{n}=-\omega_{0}<0 .
$$


Proof. Let $(\lambda, \phi)=\left(\lambda_{n}, \phi_{n}\right)$ in (7), where $\phi_{n}$ is defined by (9). Multiplying $\overline{\phi_{n}}$ on both sides of the first equation in (7) and integrating from 0 to 1 with respect to $x$, we obtain

$$
\lambda_{n}^{2} \int_{0}^{1} \rho(x)\left|\phi_{n}(x)\right|^{2} d x+\int_{0}^{1} E I(x)\left|\phi_{n}^{\prime \prime}(x)\right|^{2} d x+k \lambda_{n}\left|\phi_{n}(1)\right|^{2}=0 .
$$

Since $\operatorname{Im} \lambda_{n} \neq 0$ for sufficiently large $n$, we have, from the above equation, that

$$
2 \operatorname{Re} \lambda_{n} \int_{0}^{1} \rho(x)\left|\phi_{n}(x)\right|^{2} d x=-k\left|\phi_{n}(1)\right|^{2} \text { as } n \rightarrow \infty .
$$

Then by (9) and the Riemann-Lebesgue lemma, we have

$$
\lim _{n \rightarrow \infty}\left|\phi_{n}(1)\right|^{2}=16 e^{-\frac{1}{2} \int_{0}^{1} a(\tau) d \tau}, \lim _{n \rightarrow \infty} \int_{0}^{1} \rho(x)\left|\phi_{n}(x)\right|^{2} d x=4 \int_{0}^{1} \rho(x) e^{-\frac{1}{2} \int_{0}^{z} a(\tau) d \tau} d x,
$$

where $z=z(x)$ is specified by (29). Hence

$$
\lim _{n \rightarrow \infty} \operatorname{Re} \lambda_{n}=-2 k \frac{e^{-\frac{1}{2} \int_{0}^{1} a(\tau) d \tau}}{\int_{0}^{1} \rho(x) e^{-\frac{1}{2} \int_{0}^{z} a(\tau) d \tau} d x}<0 .
$$

The result follows.

Proof of Theorem 2.5. By Lemma 3.5 and the spectrum-determined growth condition, we need only show that

$$
\operatorname{Re} \lambda<0 \text { for any } \lambda \in \sigma(\mathcal{A}) \text {. }
$$

Since the system is dissipative, $\operatorname{Re} \lambda \leq 0$ for any $\lambda \in \sigma(\mathcal{A})$. Suppose that $\operatorname{Re} \lambda=0$. Then from $\operatorname{Re}\langle\mathcal{A} Y, Y\rangle=-k|\phi(1)|^{2}$ for each $Y=(\phi, \lambda \phi)$, we have $\phi(1)=0$. In this case, (7) becomes

$$
\left\{\begin{array}{l}
\lambda^{2} \rho(x) \phi(x)+\left(E I(x) \phi^{\prime \prime}(x)\right)^{\prime \prime}=0, \quad 0<x<1 \\
\phi(0)=\phi^{\prime}(0)=\phi^{\prime \prime}(1)=\phi^{\prime \prime \prime}(1)=\phi(1)=0
\end{array}\right.
$$

The proof is complete if we can show that there is only zero solution to (58). To this end, we follow the idea used in [17].

First, we claim that there is at least one zero of $\phi$ in $(0,1)$. In fact, by $\phi(0)=$ $\phi(1)=0$, it follows from Rolle's theorem that there is a $\xi_{1} \in(0,1)$ such that $\phi^{\prime}\left(\xi_{1}\right)=0$, which, together with $\phi^{\prime}(0)=0$, claims that $\left(E I \phi^{\prime \prime}\right)\left(\xi_{2}\right)=0$ for some $\xi_{2} \in\left(0, \xi_{1}\right)$, and so $\left(E I \phi^{\prime \prime}\right)^{\prime}\left(\xi_{3}\right)=0$ for some $\xi_{3} \in\left(\xi_{2}, 1\right)$ by the condition $\left(E I \phi^{\prime \prime}\right)(1)=0$. Hence there is a $\xi_{4} \in\left(\xi_{3}, 1\right)$ such that $\left(E I \phi^{\prime \prime}\right)^{\prime \prime}\left(\xi_{4}\right)=0$ by the condition $\left(E I \phi^{\prime \prime}\right)^{\prime}(1)=0$. However, $\left(E I \phi^{\prime \prime}\right)^{\prime \prime}\left(\xi_{4}\right)=-\lambda^{2} \rho(\xi) \phi\left(\xi_{4}\right)$; we conclude that $\phi\left(\xi_{4}\right)=0$.

Next, we show that if there are $n$ different zeros of $\phi$ in $(0,1)$, then there are at least $n+1$ number of different zeros of $\phi$ in $(0,1)$.

Indeed, suppose that

$$
0<\xi_{1}<\xi_{2}<\cdots<\xi_{n}<1, \phi\left(\xi_{i}\right)=0, i=1,2, \ldots, n .
$$

Since $\phi(0)=\phi(1)=0$, it follows from Rolle's theorem that there are $\eta_{i}, i=1,2, \ldots, n+$ 1 ,

$$
0<\eta_{1}<\xi_{1}<\eta_{2}<\xi_{2}<\cdots<\xi_{n}<\eta_{n+1}<1
$$


such that $\phi^{\prime}\left(\eta_{i}\right)=0$. Noting that $\phi^{\prime}(0)=0$, there are $\alpha_{i}, i=1,2, \ldots, n+1$,

$$
0<\alpha_{1}<\eta_{1}<\alpha_{2}<\eta_{2}<\cdots<\alpha_{n+1}<\eta_{n+1}<1
$$

such that $\left(E I \phi^{\prime \prime}\right)\left(\alpha_{i}\right)=0$. Since $\left(E I \phi^{\prime \prime}\right)(1)=0$, using Rolle's theorem again, we have $\beta_{i}, i=1,2, \ldots, n+1$,

$$
0<\alpha_{1}<\beta_{1}<\alpha_{2}<\cdots<\alpha_{n+1}<\beta_{n+1}<1
$$

such that $\left(E I \phi^{\prime \prime}\right)^{\prime}\left(\beta_{i}\right)=0$. Finally, by the condition $\left(E I \phi^{\prime \prime}\right)^{\prime}(1)=0$, we have $\vartheta_{i}, i=$ $1,2, \ldots, n+1$,

$$
0<\beta_{1}<\vartheta_{1}<\beta_{2}<\cdots<\beta_{n+1}<\vartheta_{n+1}<1
$$

such that $\left(E I \phi^{\prime \prime}\right)^{\prime \prime}\left(\vartheta_{i}\right)=0$. Therefore,

$$
\phi\left(\vartheta_{i}\right)=0, i=1,2, \ldots, n+1 .
$$

Third, by mathematical induction, there is an infinite number of different zeros $\left\{x_{i}\right\}_{1}^{\infty}$ of $\phi$ in $(0,1)$. Let $x_{0} \in[0,1]$ be an accumulation point of $\left\{x_{i}\right\}_{1}^{\infty}$. It is obvious that

$$
\phi^{(i)}\left(x_{0}\right)=0, \quad i=0,1,2,3 .
$$

Note that $\phi$ satisfies the linear differential equation $\left(E I(x) \phi^{\prime \prime}(x)\right)^{\prime \prime}+\lambda^{2} \rho(x) \phi(x)=$ 0 . Therefore, $\phi \equiv 0$ by the uniqueness of the solution of linear ordinary different equations.

Remark 4. The proof of Theorem 2.5 shows that each eigenvalue of $\mathcal{A}$ must be geometrically simple. In fact, suppose that $\left(\phi_{1}, \lambda \phi_{1}\right),\left(\phi_{2}, \lambda \phi_{2}\right)$ are any two eigenfunctions of $\mathcal{A}$ corresponding to $\lambda$. Then one can choose constants $c_{1}, c_{2}$ not identical to zero simultaneously such that $\phi=c_{1} \phi_{1}+c_{2} \phi_{2}$ satisfies $\phi(1)=0$. Now $\phi$ satisfies (58), and so $\phi \equiv 0$. Hence $\phi_{1}$ and $\phi_{2}$ are linearly independent.

From previous discussions, we see that our method can be easily used to get the Riesz basis property for the following beam equation under boundary moment feedback control:

$$
\left\{\begin{array}{l}
\rho(x) y_{t t}(x, t)+\left(E I(x) y_{x x}(x, t)\right)_{x x}=0, \quad 0<x<1, t>0 \\
y(0, t)=y_{x}(0, t)=y_{x x x}(1, t)=0 \\
y_{x x}(1, t)=-k y_{x t}(1, t), \quad k>0
\end{array}\right.
$$

It should be noted that the referring Riesz basis applied with Theorem 3.3 is accomplished by collecting all eigenfunctions of the following conservative free system:

$$
\left\{\begin{array}{l}
\rho(x) y_{t t}(x, t)+\left(E I(x) y_{x x}(x, t)\right)_{x x}=0, \quad 0<x<1, t>0 \\
y(0, t)=y_{x}(0, t)=y_{x x x}(1, t)=y_{x t}(1, t)=0 .
\end{array}\right.
$$

This is the same as that of the uniform case [1]. Moreover, the analysis in this paper shows that the low order perturbations do not affect the basis property. For example, if we assume $b(x) \in C^{3}[0,1]$, then Theorem 2.7 is still valid for the following system:

$$
\left\{\begin{array}{l}
\rho(x) y_{t t}(x, t)+b(x) y_{x x x}(x, t)+\left(E I(x) y_{x x}(x, t)\right)_{x x}=0, \quad 0<x<1, t>0 \\
y(0, t)=y_{x}(0, t)=y_{x x}(1, t)=0,\left(E I(x) y_{x x}\right)_{x}(1, t)=k y_{t}(1, t)
\end{array}\right.
$$

Let us turn to system (12). First, we give a short proof of Theorem 2.7 by virtue of Theorem 3.3. 
Proof of Theorem 2.7. Obviously, $A+B$ is a discrete operator in $H$. Write $A+B=A_{s}+T$, where $A_{s} \phi_{n}=\lambda_{n} \phi_{n}$ for all $n \geq 1$ and $T$ is a linear bounded operator on $H$. We may assume without loss of generality that $\left\|\phi_{n}\right\|=1$ for all $n \geq 1$. Since $\left\{\phi_{n}\right\}_{1}^{\infty}$ is a Riesz basis, there is a $K>0$ such that for any $\phi=\sum_{n=1}^{\infty} a_{n} \phi_{n}$ and any complex series $\left\{\beta_{n}\right\},\left|\beta_{n}\right| \leq 1$,

$$
\left\|\sum_{n=1}^{\infty} \beta_{n} a_{n} \phi_{n}\right\| \leq K\|\phi\| .
$$

By (15), we have $d_{n} \rightarrow \infty$ as $n \rightarrow \infty$. Hence for any $C>K\|T\|$, there is an integer $M>N$ such that $2\|T\| K / d_{n}<1$ for all $n \geq M$ and

$$
\left|\lambda-\lambda_{m}\right| \geq C \text { for any } \lambda \text { satisfying }\left|\lambda-\lambda_{n}\right|=C, n \geq M \text {. }
$$

First, for any $\phi=\sum_{n=1}^{\infty} a_{n} \phi_{n}$ and $\lambda$ satisfying $\left|\lambda-\lambda_{n}\right|=C, n \geq M$,

$$
\left\|C R\left(\lambda, A_{s}\right) \phi\right\|=\left\|\sum_{n=1}^{\infty} \frac{C}{\lambda-\lambda_{n}} a_{n} \phi_{n}\right\| \leq K\|\phi\|,
$$

and so $\left\|R\left(\lambda, A_{s}\right)\right\| \leq K / C$. Hence $\left\|R\left(\lambda, A_{s}\right) T\right\| \leq K\|T\| / C<1$. This shows that $\left\{\lambda|| \lambda-\lambda_{n} \mid=C, n \geq M\right\} \subset \rho\left(A_{s}+T\right)$ since $\lambda \in \sigma\left(A_{s}+T\right)$ if and only if $1 \in$ $\rho\left(R\left(\lambda, A_{s}\right) T\right)$. Let $\Gamma_{n}=\left\{\lambda|| \lambda-\lambda_{n} \mid=C\right\}, n \geq$ M. Consider eigenprojectors

$$
\begin{aligned}
Q_{n}-P_{n} & =\frac{1}{2 \pi i} \int_{\Gamma_{n}} R\left(\lambda, A_{s}+T\right) d \lambda-\frac{1}{2 \pi i} \int_{\Gamma_{n}} R\left(\lambda, A_{s}\right) d \lambda \\
& =\frac{1}{2 \pi i} \sum_{m=1}^{\infty} \int_{\Gamma_{n}}\left[R\left(\lambda, A_{s}\right) T\right]^{m} R\left(\lambda, A_{s}\right) d \lambda .
\end{aligned}
$$

One can choose $C>0$ large enough such that

$$
\left\|Q_{n}-P_{n}\right\| \leq C \sum_{m=1}^{\infty}(K\|T\| / C)^{m} K / C=K \frac{K\|T\| / C}{1-K\|T\| / C}<1 .
$$

Therefore, $\operatorname{dim}\left(Q_{n}\right)=\operatorname{dim}\left(P_{n}\right)$. Hence there exists a unique $\mu_{n},\left|\mu_{n}-\lambda_{n}\right|<C$ such that $\mu_{n} \in \sigma\left(A_{s}+T\right)=\sigma(A+B)$. This is (i). Moreover, since $\left\|P_{n} \phi_{n}\right\|=\left\|\phi_{n}\right\|=1$, we see that $Q_{n} \phi_{n} \neq 0$ and

$$
Q_{n} \phi_{n}=\phi_{n}+\frac{1}{2 \pi i} \sum_{m=1}^{\infty} \int_{\Gamma_{n}}\left[R\left(\lambda, A_{s}\right) T\right]^{m} R\left(\lambda, A_{s}\right) d \lambda d \lambda \phi_{n} .
$$

Next, take $\Lambda_{n}=\left\{\lambda|| \lambda-\lambda_{n} \mid=d_{n} / 2\right\}, n \geq M$. Then for any $\phi=\sum_{n=1}^{\infty} a_{n} \phi_{n}$ and $\lambda \in \Lambda_{n},\left\|d_{n} / 2 R\left(\lambda, A_{s}\right) \phi\right\|=\left\|\sum_{m=1}^{\infty} \frac{d_{n}}{2} \frac{1}{\lambda-\lambda_{m}} a_{m} \phi_{m}\right\| \leq K\|\phi\|$, and thus $\left\|R\left(\lambda, A_{s}\right)\right\| \leq$ $\frac{2}{d_{n}} K$. Since $\left\|R\left(\lambda, A_{s}\right) T\right\| \leq \frac{2}{d_{n}}\|T\| K<1$, we see that $\left\{\Lambda_{n}, n \geq M\right\} \subset \rho\left(A_{s}+T\right)=$ $\rho(A+B)$. Now consider

$$
\begin{aligned}
\tilde{Q}_{n}-P_{n} & =\frac{1}{2 \pi i} \int_{\Lambda_{n}} R\left(\lambda, A_{s}+T\right) d \lambda-\frac{1}{2 \pi i} \int_{\Lambda_{n}} R\left(\lambda, A_{s}\right) d \lambda \\
& =\frac{1}{2 \pi i} \sum_{m=1}^{\infty} \int_{\Lambda_{n}}\left[R\left(\lambda, A_{s}\right) T\right]^{m} R\left(\lambda, A_{s}\right) d \lambda .
\end{aligned}
$$


We have

$$
\left\|\tilde{Q}_{n}-P_{n}\right\| \leq \frac{\frac{2}{d_{n}}\|T\| K}{1-\frac{2}{d_{n}}\|T\| K}\|T\| K \leq \frac{L}{d_{n}}, \quad n \geq M,
$$

for some constant $L>0$. We may consider

$$
\left\|\tilde{Q}_{n}-P_{n}\right\| \leq \frac{L}{d_{n}}<1, \quad n \geq M .
$$

Hence $\operatorname{dim}\left(\tilde{Q}_{n}\right)=\operatorname{dim}\left(P_{n}\right)=1$, and $Q_{n}=\tilde{Q}_{n}$ as $n \geq M$. Therefore, $\psi_{n}=Q_{n} \phi_{n}$ satisfies

$$
\left\|\psi_{n}-\phi_{n}\right\|^{2} \leq L^{2} d_{n}^{-2} \text { as } n \geq M,
$$

proving the theorem. $\quad \square$

Note that the eigenproblem of (12) is to find the nonzero solution $\psi$ such that

$$
\left\{\begin{array}{l}
\mu^{2} \rho(x) \psi(x)+\mu b(x) \psi(x)+\left(E I(x) \psi^{\prime \prime}(x)\right)^{\prime \prime}=0, \quad 0<x<1 \\
\psi(0)=\psi^{\prime}(0)=\psi^{\prime \prime}(1)=0 \\
\left(E I(x) \psi^{\prime \prime}\right)^{\prime}(1)=\mu k \psi(1)
\end{array}\right.
$$

and the eigenfunction of $\mathcal{A}+\mathcal{B}$ is of the form $(\psi, \mu \psi)$.

Proof of Corollary 2.9. Let $(\mu, \psi)=\left(\mu_{n}, \psi_{n}\right)$ in (67), where $\psi_{n}$ is determined by (17). Multiplying $\overline{\psi_{n}}$ on both sides of the first equation in (67) and integrating from 0 to 1 with respect to $x$, we obtain

$\mu_{n}^{2} \int_{0}^{1} \rho(x)\left|\psi_{n}(x)\right|^{2} d x+\mu_{n} \int_{0}^{1} b(x)\left|\psi_{n}(x)\right|^{2} d x+\int_{0}^{1} E I(x)\left|\psi_{n}^{\prime \prime}(x)\right|^{2} d x+k \lambda_{n}\left|\psi_{n}(1)\right|^{2}=0$.

Since $\operatorname{Im} \mu_{n} \neq 0$ for sufficiently large $n$, we have, from the above equation, the following:

$$
\operatorname{Re} \mu_{n}=-\frac{1}{2} \frac{\int_{0}^{1} b(x)\left|\psi_{n}(x)\right|^{2} d x+k\left|\psi_{n}(1)\right|^{2}}{\int_{0}^{1} \rho(x)\left|\psi_{n}(x)\right|^{2} d x} \text { as } n \rightarrow \infty .
$$

It follows from (17) and (18) that $\left\|\psi_{n}-\phi_{n}\right\|_{L^{2}(0,1)} \rightarrow 0,\left\|\psi_{n}^{\prime}-\phi_{n}^{\prime}\right\|_{L^{2}(0,1)} \rightarrow 0$ as $n \rightarrow \infty$. By the trace theorem $\left|\psi_{n}(1)-\phi_{n}(1)\right| \rightarrow 0$. Therefore,

$$
\operatorname{Re} \mu_{n} \rightarrow-\frac{1}{2} \frac{\int_{0}^{1} b(x)\left|\phi_{n}(x)\right|^{2} d x+k\left|\phi_{n}(1)\right|^{2}}{\int_{0}^{1} \rho(x)\left|\phi_{n}(x)\right|^{2} d x} \text { as } n \rightarrow \infty .
$$

Similar to the proof of Lemma 3.5, we obtain (19).

Proof of Example 3. It follows from the proof of Corollary 2.9 that for any eigenfunction $(\psi, \mu \psi)$ of $\mathcal{A}+\mathcal{B}$

$$
\mu^{2} \int_{0}^{1}|\psi(x)|^{2} d x+\mu \int_{0}^{1} b(x)|\psi(x)|^{2} d x+\int_{0}^{1}\left|\psi^{\prime \prime}(x)\right|^{2} d x=0 .
$$

If $\operatorname{Im} \mu=0$, then from the above equation

$$
(\operatorname{Re} \mu)^{2} \int_{0}^{1}|\psi(x)|^{2} d x+\operatorname{Re} \mu \int_{0}^{1} b(x)|\psi(x)|^{2} d x+\int_{0}^{1}\left|\psi^{\prime \prime}(x)\right|^{2} d x=0 .
$$


Hence $\operatorname{Re} \mu<0$. If $\operatorname{Im} \mu \neq 0$,

$$
\operatorname{Re} \mu=-\frac{1}{2} \frac{\int_{0}^{1} b(x)|\psi(x)|^{2} d x}{\int_{0}^{1}|\psi(x)|^{2} d x} \leq-\frac{1}{2} \frac{b_{0} \int_{a}^{b}|\psi(x)|^{2} d x}{\int_{0}^{1}|\psi(x)|^{2} d x}<0 .
$$

Therefore, for any $\mu \in \sigma(\mathcal{A}+\mathcal{B}), \operatorname{Re} \mu<0$. This, together with (20), gives the exponential stability of system (22), which is indicated in [18]. By similar reasoning, when $k=0, b(x) \geq 0$ for $x \in[0,1]$ and $b(x)>b_{0}>0$ for all $x$ in some subset $(a, b) \subset[0,1]$, system $(12)$ is also exponential stable.

Finally, we give the proof of Proposition 2.10. The validity of Proposition 2.10 deduces Lemma 3.5 automatically.

Proof of Proposition 2.10. Like the transformation from (7) to (32), (67) can be transformed into

$$
\left\{\begin{array}{l}
g^{(4)}(z)+a_{1}(z) g^{\prime \prime}(z)+a_{2}(z) g^{\prime}(z)+a_{3}(z) g(z)+\lambda h^{4} \tilde{b}(z) g(z)+\lambda^{2} h^{4} g(z)=0, \\
g(0)=g^{\prime}(0)=0 \\
g^{\prime \prime}(1)=a_{11} g^{\prime}(1)+a_{12} g(1) \\
g^{\prime \prime \prime}(1)=a_{21} g^{\prime}(1)+\left[\lambda \frac{k h^{3}}{E I(1)}\left(\frac{\rho(1)}{E I(1)}\right)^{-3 / 4}+a_{22}\right] g(1)
\end{array}\right.
$$

where the functions are the same as those in (32). By Theorem 2.8 and Corollary 2.9, all eigenvalues of $\mathcal{A}+\mathcal{B}$ with sufficiently large modulus must be located on the left complex plane under the assumption (23). Following [4], by noticing the smooth assumption (3) and (23), we know that for $\lambda=\rho^{2} / h^{2},|\rho|$ sufficiently large,

$$
g^{(4)}(z)+a_{1}(z) g^{\prime \prime}(z)+a_{2}(z) g^{\prime}(z)+a_{3}(z) g(z)+\lambda h^{4} \tilde{b}(z)+\lambda^{2} h^{4} g(z)=0
$$

admits four linearly independent solutions $g_{k}, k=1,2,3,4$ for any $\rho \in S$, which satisfy

$$
\left\{\begin{array}{l}
g_{k}(z)=e^{\rho \omega_{k} z}\left[1+\frac{L_{k}(z)}{\rho}+\mathcal{O}\left(\frac{1}{\rho^{2}}\right)\right], \\
g_{k}^{\prime}(z)=\rho \omega_{k} e^{\rho \omega_{k} z}\left[1+\frac{L_{k}(z)}{\rho}+\mathcal{O}\left(\frac{1}{\rho^{2}}\right)\right], \\
g_{k}^{\prime \prime}(z)=\left(\rho \omega_{k}\right)^{2} e^{\rho \omega_{k} z}\left[1+\frac{L_{k}(z)}{\rho}+\mathcal{O}\left(\frac{1}{\rho^{2}}\right)\right], \\
g_{k}^{\prime \prime \prime}(z)=\left(\rho \omega_{k}\right)^{3} e^{\rho \omega_{k} z}\left[1+\frac{L_{k}(z)}{\rho}+\mathcal{O}\left(\frac{1}{\rho^{2}}\right)\right], \quad k=1,2,3,4,
\end{array}\right.
$$

where

$$
L_{k}(z)=-\frac{1}{4 \omega_{k}} \int_{0}^{z} a_{1}(\tau) d \tau+\frac{h^{2}}{4} \omega_{k} \int_{0}^{z} \tilde{b}(\tau) d \tau .
$$

Similar to (37)-(40), by noting (71), we can write the characteristic equation (42) as

$$
\operatorname{det}\left(\begin{array}{cccc}
1 & 1 & e^{\rho \omega_{2}} & 0 \\
\omega_{1} & \omega_{2} & -\omega_{2} e^{\rho \omega_{2}} & 0 \\
0 & \omega_{2}^{2} e^{\rho \omega_{2}}\left[1+\frac{\ell_{2}}{\rho}\right] & \omega_{2}^{2}\left[1+\frac{\ell_{3}}{\rho}\right] & \omega_{1}^{2}\left[1+\frac{\ell_{4}}{\rho}\right] \\
0 & \omega_{2}^{3} e^{\rho \omega_{2}}\left[1+\frac{\ell_{2}}{\rho}\right]-\frac{\tilde{k}}{\rho} e^{\rho \omega_{2}} & -\omega_{2}^{3}\left[1+\frac{\ell_{3}}{\rho}\right]-\frac{\tilde{k}}{\rho} & -\omega_{1}^{3}\left[1+\frac{\ell_{4}}{\rho}\right]-\frac{\tilde{k}}{\rho}
\end{array}\right)=\mathcal{O}\left(\frac{1}{\rho^{2}}\right),
$$

where $\ell_{k}=L_{k}(1)$. A direct computation yields

$$
e^{2 \rho \omega_{2}}=-1+2 \frac{\tilde{k}}{\rho} \omega_{2}+\frac{2 \ell_{2}}{\rho}+\mathcal{O}\left(\frac{1}{\rho^{2}}\right) .
$$


Substituting $\rho=-(n+1 / 2) \pi \omega_{2}+\mathcal{O}\left(n^{-1}\right)$ into (74), the term $\mathcal{O}\left(n^{-1}\right)$ satisfies

$$
-2 \omega_{2} \mathcal{O}\left(n^{-1}\right)=\frac{2 \tilde{k}}{(n+1 / 2) \pi}-\frac{2 \ell_{2}}{(n+1 / 2) \pi \omega_{2}}+\mathcal{O}\left(n^{-2}\right)
$$

hence

$$
\mathcal{O}\left(n^{-1}\right)=\frac{\tilde{k}}{(n+1 / 2) \pi \omega_{2}}-\frac{2 \ell_{2}}{(n+1 / 2) \pi \omega_{2}} \frac{1}{2 \omega_{2}}+\mathcal{O}\left(n^{-2}\right)
$$

Therefore,

$$
\rho=-(n+1 / 2) \pi \omega_{2}+\frac{2 \tilde{k}}{2(n+1 / 2) \pi \omega_{2}}+\frac{2 \ell_{2}}{(n+1 / 2) \pi \omega_{2}} \frac{1}{2 \omega_{2}}+\mathcal{O}\left(n^{-2}\right),
$$

which produces

$$
\lambda h^{2}=\rho^{2}=-2 \tilde{k}+i[(n+1 / 2) \pi]^{2}-\frac{2 \ell_{2}}{\omega_{2}}+\mathcal{O}\left(n^{-1}\right) .
$$

The required result then follows.

It is seen that Proposition 2.10 coincides with the estimates in [1] for the uniform system (1) with $k_{1}=0, b=0$.

Thus, from (24), condition (23) can be replaced by

$$
\int_{0}^{1} \tilde{b}(z) d z=\frac{1}{h} \int_{0}^{1} \frac{b(x)}{\rho(x)}\left(\frac{\rho(x)}{E I(x)}\right)^{1 / 4} d x>0 .
$$

For the case of $E I=\rho=1$, the result can be found in [6].

\section{REFERENCES}

[1] B.-Z. GUO AND R. YU, On Riesz basis property of discrete operators with application to an Euler-Bernoulli beam equation with boundary linear feedback control, IMA J. Math. Control Inform., 18 (2001), pp. 241-251.

[2] B.-Z. Guo, Riesz basis approach to the stabilization of a flexible beam with a tip mass, SIAM J. Control Optim., 39 (2001), pp. 1736-1747.

[3] B.-Z. Guo And K. Y. Chan, Riesz basis generation, eigenvalues distribution, and exponential stability for an Euler-Bernoulli beam with joint feedback control, Rev. Mat. Complut., 14 (2001), pp. 205-229.

[4] M. A. Naimark, Linear Differential Operators, Vol. I, Ungar, New York, 1967.

[5] G. D. BIRKhOFF, On the asymptotic character of the solutions of certain linear differential equations containing a parameter, Trans. Amer. Math. Soc., 9 (1908), pp. 219-231.

[6] H. Wang And G. Chen, Asymptotic locations of eigenfrequencies of Euler-Bernoulli beam with nonhomogeneous structural and viscous damping coefficients, SIAM J. Control Optim., 29 (1991), pp. 347-367.

[7] B. P. RAO, Optimal energy decay rate in a damped Rayleigh beam, in Optimization Methods in Partial Differential Equations, Contemp. Math. 209, S. Cox and I. Lasiecka, eds., AMS, Providence, RI, 1997, pp. 211-229.

[8] S. Li, J. YU, Z. LiANG, AND G. ZHU, Stabilization of high eigenfrequencies of a beam equation with generalized viscous damping, SIAM J. Control Optim., 37 (1999), pp. 1767-1779.

[9] S. Cox and E. Zuazua, The rate at which energy decays in a damped string, Comm. Partial Differential Equations, 19 (1994), pp. 213-243.

[10] A. Shubov, Basis property of eigenfunctions of nonselfadjoint operator pencils generated by the equation of nonhomogenerous damped string, Integral Equations Operator Theory, 25 (1996), pp. 289-328. 
[11] M. A. Shubov, Spectral operators generated by damped hyperbolic equations, Integral Equations Operator Theory, 28 (1997), pp. 358-372.

[12] F. Conrad And Ö. MorgüL, On the stabilization of a flexible beam with a tip mass, SIAM J. Control Optim., 36 (1998), pp. 1962-1986.

[13] G. Chen, S. A. Fulling, F. J. Narcowich, and C. QI, An asymptotic average decay rate for the wave equation with variable coefficient viscous damping, SIAM J. Appl. Math., 50 (1990), pp. 1341-1347.

[14] B. R. LI, The perturbation theory of a class of linear operators with applications, Acta Math. Sinica, 21 (1978), pp. 206-222 (in Chinese).

[15] A. G. Ramm, On the basis property for the root vectors of some nonselfadjoint operators, J. Math. Anal. Appl., 80 (1981), pp. 57-66.

[16] T. Kato, Perturbation Theory for Linear Operators, Springer-Verlag, Berlin, 1966.

[17] D. X. Feng And G. T. ZhU, The spectral property of the system operator arising from a class of elastic vibration problems, Sciences Bulletin Ser. A, 24 (1981), pp. 1473-1475 (in Chinese).

[18] G. Chen, S. A. Fulling, F. J. Narcowich, and S. Sun, Exponential decay of energy of evolution equations with locally distributed damping, SIAM J. Appl. Math., 51 (1991), pp. 266-301. 